\title{
Tangent Vectors on Tangent Euclidean Spaces
}

\author{
Open Mathematics Collaboration*广
}

May 28, 2021

\begin{abstract}
This is an article on differential geometry that connects tangent vectors and tangent Euclidean spaces [1].
\end{abstract}

keywords: differential geometry, Euclidean space, tangent vector, tangent space

The most updated version of this white paper is available at https://osf .io/vpz9x/download

\section{The tangent space in each point of $\mathbb{R}^{3}$ is $\mathbb{R}^{3}$}

1. In the following, for the sake of simplicity, sometimes we will omit the words "let", "consider", etc, in which is implicitly assumed hereafter.

\section{Tangent vector}

2. The set of all ordered triples of real numbers $\mathbf{p}=\left(p_{1}, p_{2}, p_{3}\right)$, called points, is a Euclidean 3-space $\mathbb{R}^{3}$.

*All authors with their affiliations appear at the end of this white paper.

${ }^{\dagger}$ Corresponding author: mplobo@uft.edu.br| Open Mathematics Collaboration 
3. $\mathbf{p}$ and $\mathbf{v}$ are points of $\mathbb{R}^{3}$.

4. $\mathbb{R}^{3}$ is a vector space over the real numbers.

5. So, $\mathbf{p}$ and $\mathbf{v}$ are also vectors.

6. Let $\mathbf{p}$ be the starting point and $\mathbf{p}+\mathbf{v}$ the end point.

7. $\mathbf{v}$ is a vector called the change of $\mathbf{p}$.

8. Let's call $\mathbf{p}$ its point of application and $\mathbf{v}$ the vector part.

9. $\mathbf{v}_{p}$ is a tangent vector to $\mathbb{R}^{3}$ if it consists of two points (vectors), $\mathbf{p}$ and $\mathbf{v}$.

10. An example of $(9)$ is: $\mathbf{p}=(\mathbf{0}, \mathbf{0}, \mathbf{1}), \mathbf{v}=(\mathbf{0}, \mathbf{1}, \mathbf{1})$ and $\mathbf{v}_{\mathbf{p}}=(\mathbf{0}, \mathbf{1}, \mathbf{2})$.

11. In (10), $\mathbf{v}_{\mathbf{p}}$ consists of $\mathbf{p}$ and $\mathbf{v}$ because $\mathbf{v}_{\mathbf{p}}=\mathbf{p}+\mathbf{v}$.

\section{Tangent space}

12. Consider $T_{p}$ as the set of all tangent vectors having $\mathbf{p}$ as a point of application.

13. Then $T_{p}$ is the tangent space of $\mathbb{R}^{3}$ at $\mathbf{p}$.

14. The tangent space in each point of $\mathbb{R}^{3}$ is $\mathbb{R}^{3}$.

\section{Final Remarks}

15. This article connected the concept of a tangent vector with the concept of a tangent Euclidean space.

16. It has a pure mathematical application here [2]. 


\section{Open Invitation}

Review, add content, and co-author this white paper [3,4].

Join the Open Mathematics Collaboration.

Send your contribution to mplobo@uft.edu.br.

\section{Open Science}

The latex file for this white paper together with other supplementary files are available in [5].

\section{How to cite this paper?}

https://doi.org/10.31219/osf.io/vpz9x

\section{Acknowledgements}

+ Open Science Framework

https://osf.io

\section{Agreement}

All authors agree with [4].

\section{References}

[1] O'neill, Barrett. Elementary differential geometry. Elsevier, 2006.

[2] Lobo, Matheus P. "The Metric Tensor Pullback." OSF Preprints, 14 May 2019. https://doi.org/10.31219/osf.io/puhzw

[3] Lobo, Matheus P. "Microarticles." OSF Preprints, 28 Oct. 2019. https://doi.org/10.31219/osf.io/ejrct 
[4] Lobo, Matheus P. "Simple Guidelines for Authors: Open Journal of Mathematics and Physics." OSF Preprints, 15 Nov. 2019.

https://doi.org/10.31219/osf.io/fk836

[5] Lobo, Matheus P. "Open Journal of Mathematics and Physics (OJMP)." OSF, 21 Apr. 2020. https://osf .io/6hzyp/files

\section{The Open Mathematics Collaboration}

Matheus Pereira Lobo (lead author, mplobo@uft.edu.br) ${ }^{1}$

https://orcid.org/0000-0003-4554-1372

Shirlei Nabarrete Dezidério ${ }^{1}$

${ }^{1}$ Federal University of Tocantins (Brazil) 